\title{
Comparison of B-Spline and Zernike fitting techniques in complex wavefront surfaces
}

\author{
M.Ares, S.Royo, J.Caum, C.Pizarro \\ Center for Sensor, Instrumentation and System Development, Technical University of Catalunya \\ (CD6-UPC) \\ Rambla Sant Nebridi 10,08222 Terrassa Spain
}

\begin{abstract}
Zernike polynomial fitting has been the commonplace alternative for assigning a measured wavefront a given shape. However, Zernike polynomials have intrinsic limitations under given conditions, mainly in complex wavefronts with for instance decentered double-peaks or with relevant undulations. The main goal of this paper is analyzing an alternative to Zernike fitting based in B-Spline fitting, comparing the strengths and weaknesses of each representation when applied to wavefront fitting. Simple and complex wavefront cases will be presented and studied, and the quality of their fitted representations using Zernike and B-Spline polynomials will be compared, presenting the main factors relevant in their comparison. Moreover, the case of random white noise added to the estimated data will allow an insight into the expected behavior of both representations when applied to experimental data.
\end{abstract}

Keywords: Wavefront reconstruction, Wavefront fitting, Zernike polynomials, B-Spline.

\section{INTRODUCTION}

The last step in the optical wavefront sensing train is the reconstruction of the wavefront surface from sampled measurements that have been taken under different techniques and conditions ${ }^{1}$. Besides the different sources of error related to the measurement procedure used, there is always a final error that arises during wavefront reconstruction. This fitting error describes how far the reconstructed wavefront from the originally measured data is, and it is usually calculated as the root mean square (rms) fit deviation ${ }^{2}$. Basically, reconstruction algorithms can be categorized as being either zonal or modal ${ }^{3}$. The modal approach involves data approximation in the whole domain by a polynomial function, whereas in the zonal model each reconstructed wavefront value is estimated from only their spatial neighbors data.

From the early 90's modal Zernike polynomial representation has been commonly chosen for this purpose. It presents a serie of advantages, from its simple analytical form, to the orthogonality of the Zernike polynomial basis in a continuous domain, not forgetting its low order terms are easily related to the classical Seidel aberrations. However, Zernike polynomials have intrinsic limitations when dealing with complex wavefronts. It has been shown how surfaces with multi-peak and multi-hole shape require flexible zonal approaches to get a satisfactory reconstruction result ${ }^{4}$. Such surfaces have, up to date, not been too relevant in optical testing with the exception of top-level astronomical applications, mainly in adaptive optics (AO).

Nowadays, complex wavefront generation from new optical elements such as deformable mirrors ${ }^{5}$, liquid-crystal spatial light modulators, and, more recently, programmable phase modulators ${ }^{6}$, is allowed. Local shape changes can be generated over the entire 2D domain to widely extend the classical AO applications to general optical testing, allowing a real local control on the shape of incoming or outcoming wavefronts in different applications. The interest of analyzing alternatives for proper fitting of the complex wavefront shapes introduced in the optical bench by the abovementioned units becomes evident.

A well-suited basis for this purposes are B-Spline polynomials. From our knowledge, they have been very rarely used in wavefront description, we guess due to the existence of a simple basis performing well for "normal" optical wavefronts, and to their mathematical complexity for beginners. However, B-Splines have the advantage of being intrinsically zonal, of being fitted to the wavefront through well-known least-squares procedures, and of having a number of parameters allowing the control of its smoothness and degree of detail. Moreover, they are a commonly used description for industrial surfaces, as far as computerized numerically controlled (CNC) tools are easily commanded using this type of

5856-76 V. 1 (p.1 of 10) / Color: No / Format: A4 / Date: 2005-03-29 06:31:26 
surface description. For instance, ophthalmic lens manufacturers use CNC systems in the generation of different aspheric or progressive addition optical surfaces, although wavefront control is not usually performed using the same basis, which obviously should be the optimum scheme for industrial production.

The goal of this paper is to present a zonal B-Spline fitting method as a good alternative to modal Zernike technique when dealing with complex wavefronts, or whenever local control parameters are needed in the wavefront description. Simple and complex wavefronts will be generated and the quality of their fitted representations using Zernike and BSpline methods will be compared.

The manuscript is organized as follows. In Section 2 a short theoretical review of both fitting techniques is presented. This walk along Zernike and B-Spline main concepts will let the reader understand their own fitting characteristics. Next, the least squares system solving by the singular value decomposition (SVD) method is introduced. Section 3 shows the simple and complex wavefront reconstruction results attained by Zernike and B-Spline techniques. From these, a final insight into simulated experimental data has been done. In Section 4, we give a summary of the main conclusions of the work.

\section{THEORETICAL BACKGROUND}

A mathematical non-rigorous review of the two analyzed fitting techniques will let us introduce their own wavefront reconstruction philosophies when dealing with discrete data. The main theoretical concepts involved in both representations are presented, to give the reader a more complete knowledge of each fitting technique. However, none of them is an intensive presentation of both polynomial bases, which would exceed too much the purposes of this paper.

\subsection{D Zernike fitting technique}

Zernike circular polynomials were developed as a convenient set for representing optical wavefronts over a circular pupil. Over their simple analytic form, which makes them more practical than other polynomial basis, Zernike expansion terms can also easily be related, in its lower orders, to classical Seidel aberrations such as spherical aberration, astigmatism and coma.

However, the main advantages of the Zernike representation are a direct consequence of the orthogonality of its basic modes over the unit circle. This means the coefficient of each of the modes is linearly independent of the remaining ones, and also that the wavefront mean square error (understood as its deviation from a spherical surface) is the sum of the mean square error of individual known modes. The lower this last value the less aberrated the wavefront is. However, orthogonality is only achieved in the theoretical case of the description of a continuous surface. When dealing with wavefronts measured in the real world only a discrete number of samples is available, so only a good approximation of the last two properties is attained.

Some different notations for the Zernike polynomials exist in the literature. In our work we follow the one described at Malacara $^{2}$. As a complete modal set, any wavefront can be expressed as a linear combination of Zernike circular polynomials as follows

$$
W(\rho, \vartheta)=\sum_{n=0}^{k} \sum_{m=0}^{n} a_{n m} \cdot Z_{n}^{l}(\rho, \vartheta)=\sum_{i=1}^{L} a_{i} \cdot Z_{i}(\rho, \vartheta) \quad, l=n-2 m
$$

where each polynomial basic function can be expressed as the product of a radial and an angular component

$$
Z_{n}^{l}(\rho, \vartheta)= \begin{cases}R_{n}^{l}(\rho) \cdot \cos (l \cdot \vartheta) & \text { for } l \leq 0 \\ R_{n}^{l}(\rho) \cdot \sin (l \cdot \vartheta) & \text { for } l>0\end{cases}
$$

where

$$
R_{n}^{i}(\rho)=R_{n}^{n-2 m}(\rho)=\sum_{s=0}^{m}(-1)^{s} \cdot \frac{(n-s) !}{s !(m-s) !(n-m-s) !} \cdot \rho^{n-2 s}
$$

with polar unit circle data coordinates $0 \leq \rho \leq 1,0 \leq \vartheta \leq 2 \pi$.

The value of the parameters involved in that description play a leading role in creating the needed Zernike fitting function. The main one is $k$, which is the Zernike polynomial degree elected, and the other ones are related to it. The $n$ value ranges from 0 to $k$-in unitary steps- fixing the degree of each radial component. For each $n$ value, $l$ varies between $-n$ and $n$ in 2 unit steps fixing the azimuthal frequency of the angular component. Thus, both parameters

5856-76 V. 1 (p.2 of 10) / Color: No / Format: A4 / Date: 2005-03-29 06:31:26 
describe each $Z_{n}^{l}$ Zernike mode. The number $L$ of Zernike modes that compose the polynomial fitting function is also related to the polynomial degree $k$, through

$$
L=(k+1) \cdot(k+2) / 2
$$

Equation (1) shows the global fitting philosophy of the Zernike representation; that is, the whole data $W$ are approximated by an unique function of degree $k$.

Given $N$ discrete data points are available from the measured wavefront, described as $W_{r}\left(\rho_{r}, \vartheta_{r}\right) \quad r=1,2, \ldots, N$,where $\left(\rho_{r}, \vartheta_{r}\right)$ are the normalized 2D polar data coordinates and $W_{r}$ the corresponding wavefront values, equation (1) unknown parameters are only the Zernike coefficients $a_{i}$. Obtaining the best estimation of those parameters means solving the linear least squares problem described by the transformation of (1) in a matrix linear problem

$$
Z \cdot a=W
$$

where $W$ is the $N \times 1$ data matrix containing the sampled wavefront values, $a$ is the $L \mathrm{x} 1$ matrix containing the Zernike coefficients, and $Z$ is the $N \times L$ matrix containing the values of the Zernike polynomials in each sampling point

$$
\left(\begin{array}{ccccc}
Z_{1}\left(\rho_{1}, \vartheta_{1}\right) & Z_{2}\left(\rho_{1}, \vartheta_{1}\right) & \ldots & \ldots & Z_{L}\left(\rho_{1}, \vartheta_{1}\right) \\
Z_{1}\left(\rho_{2}, \vartheta_{2}\right) & Z_{2}\left(\rho_{2}, \vartheta_{2}\right) & \ldots & \ldots & Z_{L}\left(\rho_{2}, \vartheta_{2}\right) \\
\vdots & \ddots & \ddots & \ddots & \vdots \\
\vdots & \ddots & \ddots & \ddots & \vdots \\
\vdots & \ddots & \ddots & \ddots & \vdots \\
\vdots & \ldots & \ddots & \ddots & \vdots \\
Z_{1}\left(\rho_{N}, \vartheta_{N}\right) & Z_{2}\left(\rho_{N}, \vartheta_{N}\right) & \ldots & \ldots & Z_{L}\left(\rho_{N}, \vartheta_{N}\right)
\end{array}\right) \cdot\left(\begin{array}{c}
a_{1} \\
a_{2} \\
\vdots \\
\vdots \\
a_{L}
\end{array}\right)=\left(\begin{array}{c}
W\left(\rho_{1}, \vartheta_{1}\right) \\
W\left(\rho_{2}, \vartheta_{2}\right) \\
\vdots \\
\vdots \\
\vdots \\
\vdots \\
W\left(\rho_{N}, \vartheta_{N}\right)
\end{array}\right)
$$

The least squares method allowing to solve equation (6) will be discussed in extent in Section 2.3.

\subsection{D B-Spline fitting technique}

In the early 70's two main techniques were formulated for allowing surface design in automobile, aircraft and naval industries $^{7}$. Both of these surface computationally-aided constructions begin with the definition of an easy-to-build polygonal model of the surface to be built. A flexible local modification of this polygonal model by changing the position of its vertices allowed designing the "smooth polygon" with the desired shape.

The first of these techniques was developed by P. E. Bézier for its use in the design of Renault automobile parts, and had a great success in industrial design environments, as its mathematical formulation is perfectly adapted to that field. However, Bézier surfaces do not really present local control, as far as any point on its surface is dependent on the values of all the vertices in the defined polygonal surface, meaning that a change in one vertex is felt throughout the entire span.

This limiting characteristic is overcome by the second of the techniques, the B-Spline method. B-Spline is a specific smooth surface generating formulation that belongs to general spline philosophy ${ }^{8}$. Any smooth, piecewise polynomial function is called a spline. The philosophy is to divide the domain plane into sufficiently small sub-areas, in such fashion that, on each sub-area, a polynomial of relatively low degree can provide a good approximation of the surface data.

Nowadays, several different implementations of the B-Spline surface model have been extensively studied. Perhaps the simplest is the tensor product one. A 3D spline can be represented by that B-Spline model as follows

$$
W(x, y)=\sum_{i=0}^{n} \sum_{j=0}^{m} a_{i j} \cdot B_{i, k}(x) \cdot B_{j, l}(y)
$$


where $a_{i j}$ are the so-called control points, acting as sub-area control parameters, and $B_{i, k}(x)$ and $B_{j, l}(y)$ are the $i t h$ and $j$ th B-Spline of degree $k-1$ and $l-1$ in $x$ and $y$ direction, respectively. Equation (7) represents a wavefront as a linear combination of B-Spline "Basic" functions. A certain formal similarity to equation (1), where the Zernike representation was applied to wavefront description may be appreciated, but no other exists as B-Spline formulation will demonstrate.

For simplicity, the discussion to follow will deal just with the univariate $x$-direction spline. No formulation difference is introduced in the 2D case, as the bivariate case is the product of two separate univariates.

Three important user-elected parameters are involved in the description of the B-Spline representation: the degree $k-1$ of B-Spline functions, the smoothness level $\mu_{s}$ at subinterval limits (where $\mu_{s}=0$ means function discontinuity, $\mu_{s}=1$ means function continuity, $\mu_{s}=2$ means function and first derivative continuity, and so on), and the number of breakpoints $N B P x$ in the interval $\left[x_{s=0}, x_{s=1}, \ldots, x_{s=N B P x-1}\right]$.

Besides real NBPx, the B-Spline formulation creates the so-called "knots" $t_{i}$. Knots are equivalent to breakpoints but, as knots, some breakpoints may be repeated with a given multiplicity. The resulting multiplicity of each knot is determined by the smoothness level following the rule:

$$
\text { knot multiplicity }=k-\mu_{s}
$$

The non-decreasing knot set is formed by all the interior breakpoints repeated knot multiplicity times, and the two endpoints of the interval repeated $k$ times. Although this last requirement can be relaxed, it has become a standard. In knot notation the set is described as $\left[t_{0}, t_{1}, t_{2}, \ldots, t_{n+k}\right]$ where $n+1$ is the number of control points, already defined in equation 7.

Taking into account the previous formulation, a practical relation lets us know the number of control points of a given B-Spline representation in terms of the 3 user-elected parameters:

$$
n+1=\left(\sum_{s=2}^{N B P x-1} k-\mu_{s}\right)+2 \cdot k-k
$$

As equation (7) shows, there are the same number of control points and B-Spline basic functions. Each control point is a weight associated to each B-Spline function, and also has a local meaning because of the B-Spline local support embedded in its own definition. The $i$ th $\mathrm{B}-$ Spline basic function of degree $k-1$ is defined by

$$
\begin{aligned}
& B_{i, k}(x)=\frac{x-t_{i}}{t_{i+k-1}-t_{i}} \cdot B_{i, k-1}(x)+\frac{t_{i+k}-x}{t_{i+k}-t_{i+1}} \cdot B_{i+1, k-1}(x) \\
& B_{i, 1}(x)= \begin{cases}1, & t_{i} \leq x \leq t_{i+1} \\
0, & \text { otherwise }\end{cases}
\end{aligned}
$$

So the $i t h$ B-Spline basic function is the sum of $k$ polynomials of degree $k-1$ over the knot subinterval $\left[t_{i} t_{i+1}, \ldots, t_{i+k}\right]$. Outside that interval the function has zero value. Oth B-Spline support is $\left[t_{0}, t_{1}, \ldots, t_{k}\right], 1$ st B-Spline support is $\left[t_{1}, t_{2}, \ldots, t_{1+k}\right]$, and so on up to $n t h$ B-Spline which is defined over $\left[t_{n}, t_{n+1}, \ldots, t_{n+k}\right]$.

Going to the particular B-Spline scheme we have applied to wavefront description, we have fixed $k=l=4$ (cubic BSpline functions) and $\mu=3$ (continuity of the polynomial pieces up to $2^{\circ}$ derivative in all interior breakpoint places). Thus, only $N B P x$ and $N B P y$ remain as free parameters. For simplicity, we have considered the same number of breakpoints $(N B P)$ in $x$ and $y$ directions and data coordinates normalized over each knot subinterval taking values from 0 to 1 following

$$
t x_{r}=\frac{x_{r}-t_{i}}{t_{i+1}-t_{i}} \quad, x_{r} \in\left[t_{i}, t_{i+1}\right]
$$

In that situation, the recursive B-Spline description (10) becomes analytical and each univariate cubic B-Spline basic function $B_{i, 4}(t x)$ with support $\left[t_{i}, t_{i+1}, \ldots, t_{i+4}\right]$ may be described as 


$$
B_{i, 4}(t x)=B_{i, 1}(t x)+B_{i+1,1}(t x)+B_{i+2,1}(t x)+B_{i+3,1}(t x)
$$

where

$$
\begin{aligned}
& B_{i, 1}(t x)=\frac{t x^{3}}{6} \\
& B_{i+1,1}(t x)=\frac{1}{6} \cdot\left(1+3 \cdot t x+3 \cdot t x^{2}-3 \cdot t x^{3}\right) \\
& B_{i+2,1}(t x)=\frac{1}{6} \cdot\left(4-6 \cdot t x^{2}+3 \cdot t x^{3}\right) \\
& B_{i+3,1}(t x)=\frac{1}{6} \cdot(1-t x)^{3}
\end{aligned} t x \in[0,1]
$$

Returning to the bivariate B-Spline scheme and given $N$ discrete experimental data points $W_{r}\left(t x_{r}, t y_{r}\right), r=1,2, \ldots, N$ are available, the unknown parameters in equation (7) are only the values of the control points $a_{i j}$. As in the case of the Zernike basis, solving the linear least squares problem described by equation (7) let us know the best control point values to describe the experimental data points obtained.

\subsection{The Singular Value Decomposition method}

The SVD of a matrix $A \in \mathfrak{R}^{m x^{n}}$ is a robust matrix decomposition method of great theoretical and practical importance for solving least squares problems ${ }^{9}$. Despite its higher computational cost $\left(m \cdot n^{2}+5 n^{3} / 3+4 n^{3}\right.$ operations) when compared with other methods such as Cholesky decomposition or QR decomposition ${ }^{10}$, SVD method can always provide an unique solution even when the matrix moves away its full rank. Moreover, SVD will also diagnose this rank deficiency, giving useful information in the shape of the condition number value.

The SVD of a data matrix $A \in \mathfrak{R}^{m \times n}$ is the matrix product ${ }^{11}$

$$
U \cdot W \cdot V^{T}
$$

where $U \in \mathfrak{R}^{m x^{n}}$ is a column orthogonal matrix, $V \in \mathfrak{R}^{n x^{n}}$ is an orthogonal matrix, and $W \in \mathfrak{R}^{n x^{n}}$ is a diagonal matrix. The diagonal elements of the last matrix are called the singular values of $A$. They have the information of data matrix rank deficiency through the so-called condition number, which is defined as the ratio of the largest to the smallest singular value. Data matrix singularity or close to the singularity occurs when the condition number is too large; i.e. if its reciprocal approaches the machine's floating-point precision. The problem is then called ill-conditioned, and SVD provides a bad least squares solution. Otherwise, the obtained solution is good.

From (14), it follows that

$$
A^{-1}=V \cdot W^{-1} \cdot U^{T}
$$

which is called the pseudoinverse of $A$. As the linear least squares problem has the well-known form $A \cdot x=b$, the SVD pseudoinverse solution is

$$
\hat{x}=A^{-1} \cdot b
$$

The quality of the solution can be expressed as an error value by $|b-A \cdot \hat{x}|$. Following that, the merit function selected to describe the quality of our fitting problems has been the rms fit deviation, that is

$$
r m s \text { fit deviation }=\frac{1}{N} \cdot \sqrt{\sum_{r=1}^{N}\left(W_{r}-\hat{W}_{r}\right)^{2}}
$$

where $N$ is the number of data points, $W_{r}$ is the original wavefront at a given data point $r$, and $\hat{W}_{r}$ is the reconstructed wavefront.

5856-76 V. 1 (p.5 of 10) / Color: No / Format: A4 / Date: 2005-03-29 06:31:26 


\section{COMPARISON OF ZERNIKE AND B-SPLINE FITTING TECHNIQUES}

Before make the fitting comparison, the number of discrete wavefront data and their spatial distribution type (regular or irregular) were studied to determine their effect in the reconstruction process. Constant rms fit deviation results under different cases both for the Zernike and B-Spline representations showed that none of these factors had any relevant influence on the quality of the fit.

In order to know the capabilities of both fitting methods when applied to different surface types, two sampled theoretical wavefronts were generated. As simple wavefront, a spherical wavefront with a radius of curvature of $100 \mathrm{~mm}$ was selected (Fig.1), while as complex wavefront we chose a decentered double-peak surface with a single hole -historically known as Franke's function ${ }^{4}$ (Fig.2). A realistic spatial sampling of 1089 equally spaced data points in a square pupil was selected for both wavefronts.

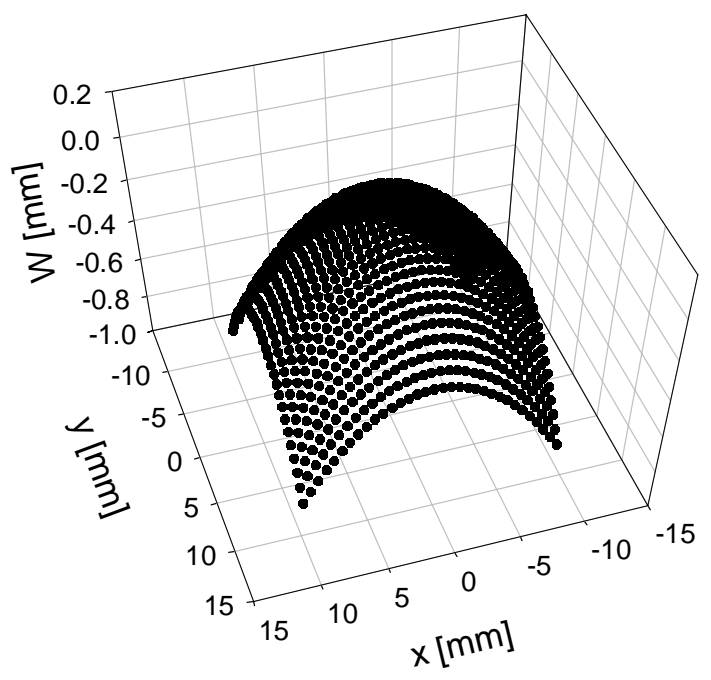

Fig. 1- Original simple wavefront

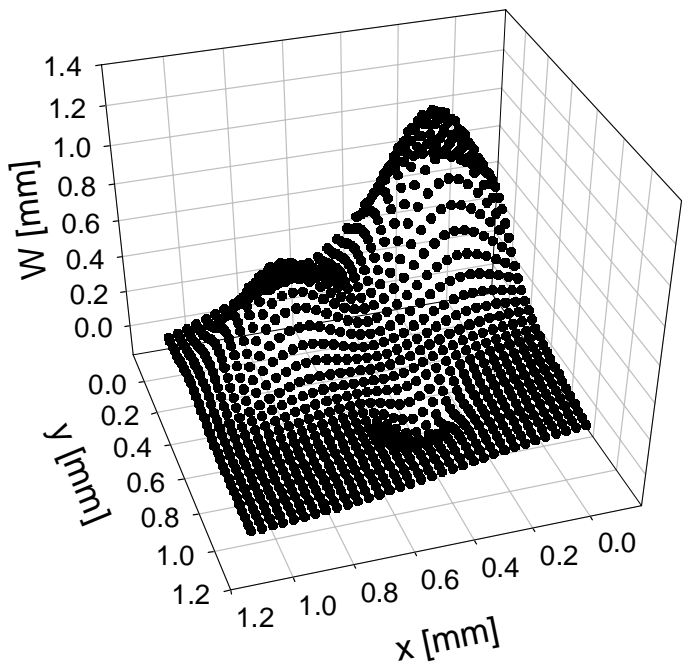

Fig. 2- Original complex wavefront

\subsection{Simple wavefront fitting analysis}

For evaluation purposes of Zernike and B-Spline representations, several polynomial degrees and number of breakpoints, respectively, were considered for the fit over both wavefronts. The comparison of the rms fit deviation as a function of those parameters for the case of the simple wavefront is depicted in Fig.3. Results show the Zernike method yields excellent fitting quality for all degree values from degree $=4$. Up to fourth degree, even with this simple wavefront the fit is not good enough. As no higher-order aberrations terms are involved in the simple case, a low degree (4 or 5 ) Zernike polynomial expansion is the optimum fitting scheme, avoiding the more time consuming computation of medium-high degree Zernike descriptions.

Cubic B-Spline representation attains a similar rms fit deviation as the Zernike basis for higher NBP values -not for lower ones-, although B-Spline calculation is more computationally intensive than the equivalent Zernike case. This makes the B-Spline representation a worse option for fitting simple wavefronts. In this sense, Fig.4 presents the comparison of the needed computational operations required for obtaining the least squares simple wavefront fitting solution under different degree/NBP values.

\subsection{Complex wavefront fitting analysis}

An equivalent study has been applied to the theoretical complex wavefront shape. The rms deviation is obtained again for different Zernike degree and B-Spline NBP conditions, as previously did for the simplest one (Fig.5). As it can be observed, the B-Spline representation yields a better representation of the complex wavefront. Very good deviation values are achieved in the B-Spline high NBP zone, not reached by any of the Zernike functions. Notice, however, the values of the rms deviation does not yield the optimum values obtained for the simple wavefront in the lower degree/NBP values. The optimum fit should then be considered the one with the lower NBP number in the asymptotic behavior. 


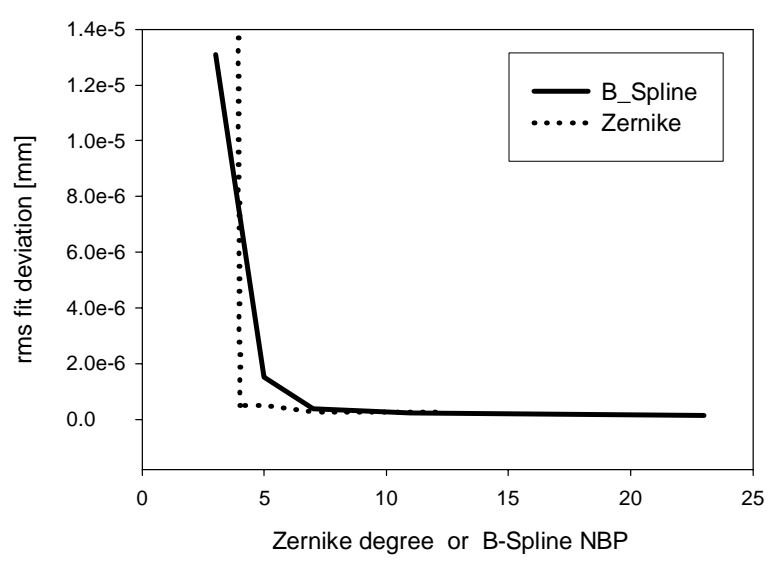

Fig. 3- Simple wavefront rms fit deviation against degree of the Zernike polynomial and NBP for B-Spline technique.

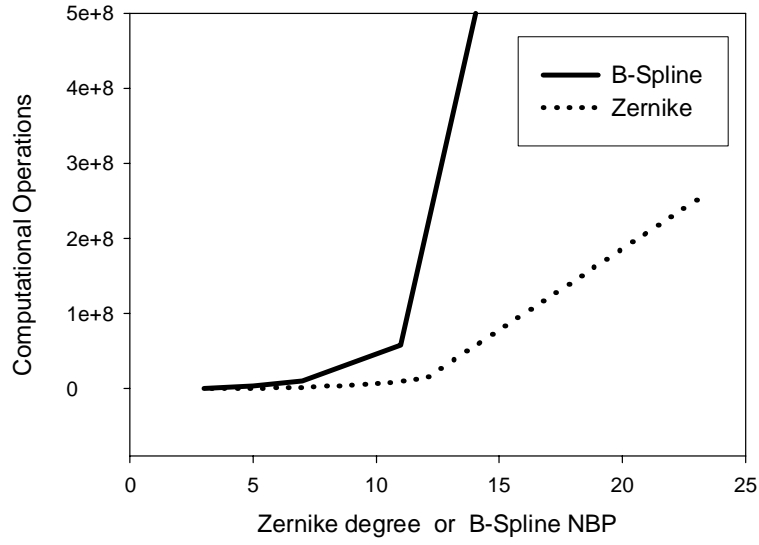

Fig. 4 -Computational operations for several Zernike and B-Spline situations.

Whereas B-Spline fitting quality increases with the NBP up to reach an almost constant value comparable with the rms attained for the simple wavefront case, Zernike fitting does not attain such asymptotic behavior at all. Even more, from a critical value of the polynomial degree the fit quality worsens noticeably. This behavior is related to the fact that the least squares problem becomes poorly conditioned at higher degree values, degrading the solution obtained. It should be stressed that this ill-conditioned problem does not occur in the B-Spline case. Fig.6 presents a comparison of the condition number value for the Zernike and B-Spline fit of the complex wavefront.

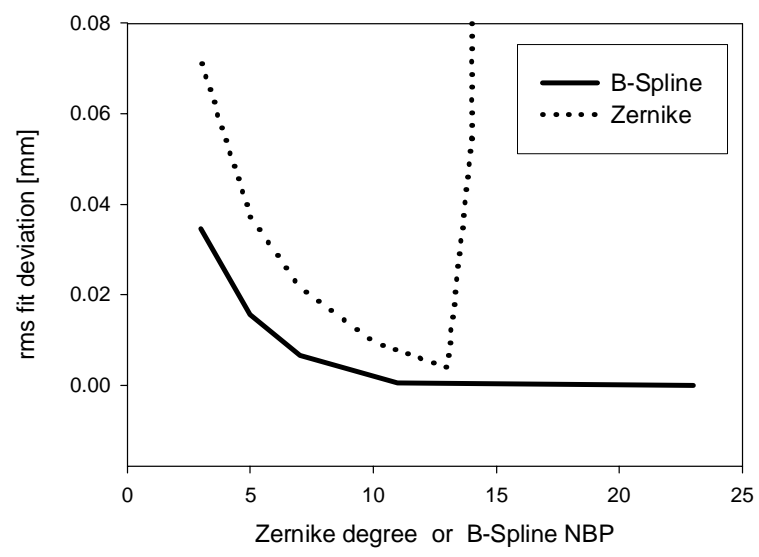

Fig. 5- Complex wavefront rms fit deviation against degree of the Zernike polynomial and NBP for B-Spline technique.

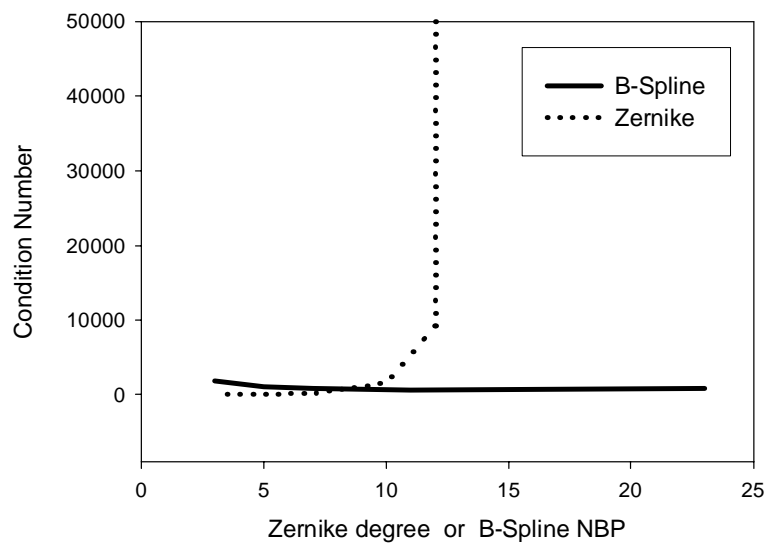

Fig. 6- Condition number for several Zernike and B-Spline situations.

\subsection{Insight into experimental cases}

Once the two fitting techniques have been analyzed in theoretical wavefronts, we now will deal with theoretical and experimental wavefronts including noise to allow an insight into its expected fitting behavior when applied to experimental data. We have generated a synthetic experimental spherical wavefront with spatial random white noise added with relative values between $\pm 1 \%$ of wavefront height peak-to-valley. From this, a Zernike polynomial fitting of degree 5 is applied, as suggested by the results in Section 3.1. A good rms deviation value of $4.903 \mathrm{e}-3 \mathrm{~mm}$. is obtained. The results of the fit are shown in Fig.7, where contour plots of the original wavefront without noise (Fig. 7a) and with 
noise added (Fig.7b) are shown. Fig. 7c presents the contour plot of the reconstructed wavefront, using a Zernike basis with degree 5 , showing the excellence of the fitting when compared with the initial surface.

In the case of this simple wavefront, the same fitting is applied to an experimental spherical wavefront, sensed using a Shack-Hartmann (SH) sensor applied to the wavefront transmitted by an spherical ophthalmic lens of $4.5 \mathrm{D}$. The experimental surface (Fig. 7d) is firstly obtained by a local integration algorithm from the measured wavefront slopes. A rms deviation of $0.804 \mathrm{e}-3 \mathrm{~mm}$ is attained in the degree-5 Zernike fit, confirming the validity of theoretical analysis in real situations. Fig. 7e shows the reconstructed surface obtained from this fit.

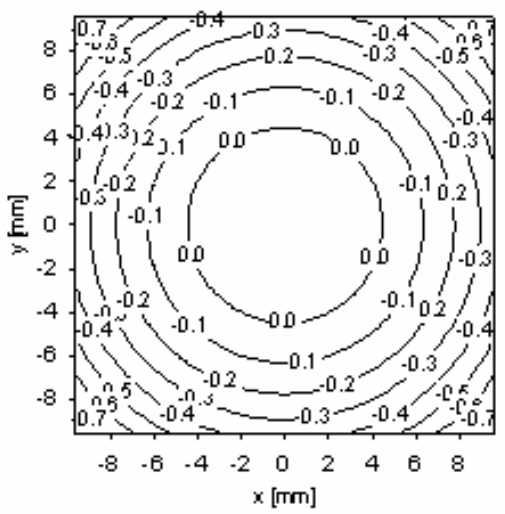

(a)

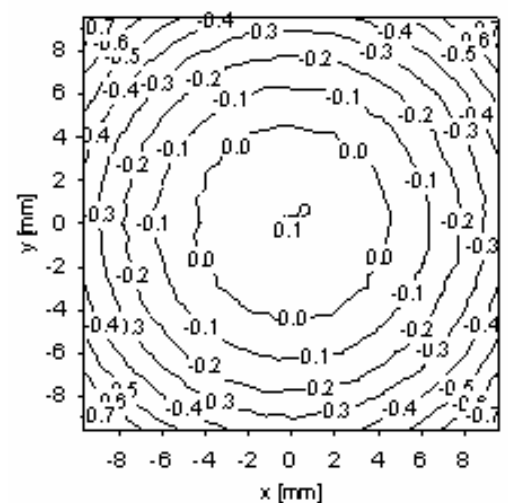

(b)

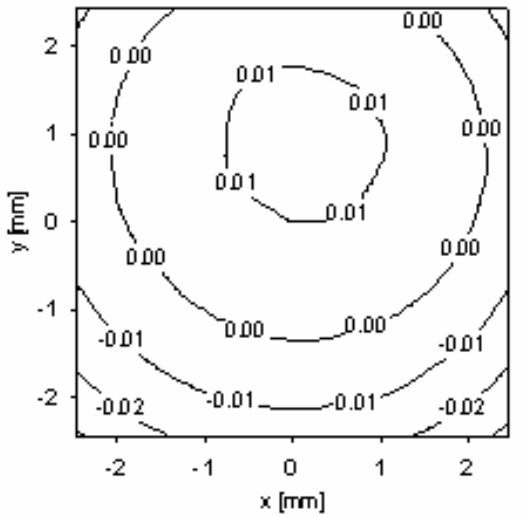

(d)

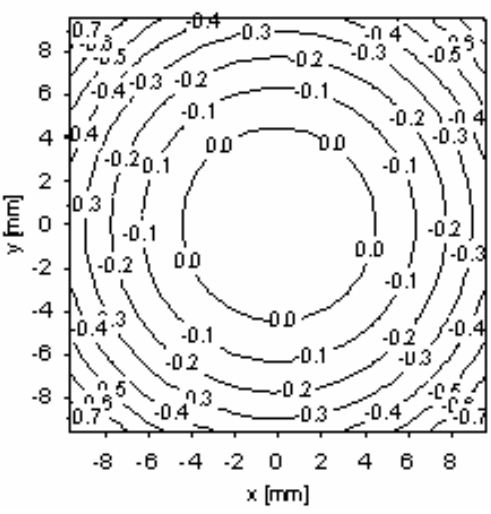

(c)

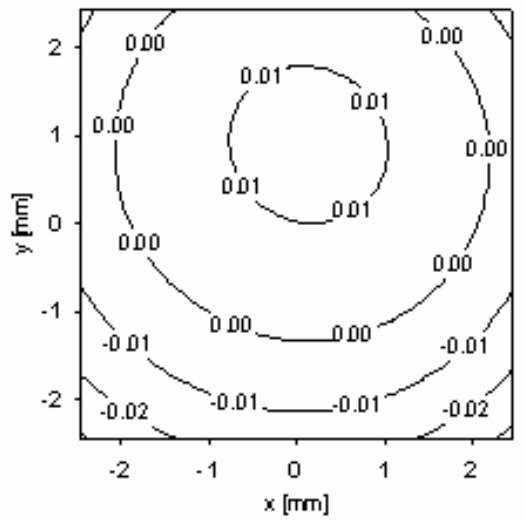

(e)

Fig.7- (a) Contour plot of the original simple wavefront, (b) contour plot of the original simple wavefront plus random noise, (c) contour plot of the reconstructed simple wavefront plus random noise by a Zernike degree $=5$ fitting, (d) contour plot of the experimental simple wavefront, (e) contour plot of the reconstructed experimental simple wavefront by a Zernike degree $=5$ fitting.

5856-76 V. 1 (p.8 of 10) / Color: No / Format: A4 / Date: 2005-03-29 06:31:26 
The same theoretical plus noise situation was also generated for the complex case, although in this case no possibility of experimental validation was available when preparing this paper. Again, a synthetic experimental complex wavefront was generated by adding a spatial random white noise of $\pm 1 \%$ of wavefront height peak-to-valley. This wavefront was fitted using an 11x11 NBPs B-Spline, placed at the start of the asymptotic behavior in Fig.5, following the conclusions of Section 3.2. As expected, a nice fit was obtained, with a $4.553 \mathrm{e}-3 \mathrm{~mm}$. rms value obtained, of the same order of the degree-5 Zernike fit for the simple wavefront case. Fig.8 shows the contour plot curves for the theoretical, noisy and fitted wavefronts. An additional result worth mentioning is that the use of a very high number of breakpoints allows the B-Spline representation even to follow the noise contributions, which may not be desirable in some cases. This confirms our conclusion of selecting NBP numbers as small as possible but within the rms tolerance desired for the fit, or at least in the beginning of the described asymptotic behavior of rms in Fig.5. Results for the experimental case in complex wavefronts are expected to follow those of the synthetic wavefronts, as in the case of the simple wavefronts presented in Fig.7.
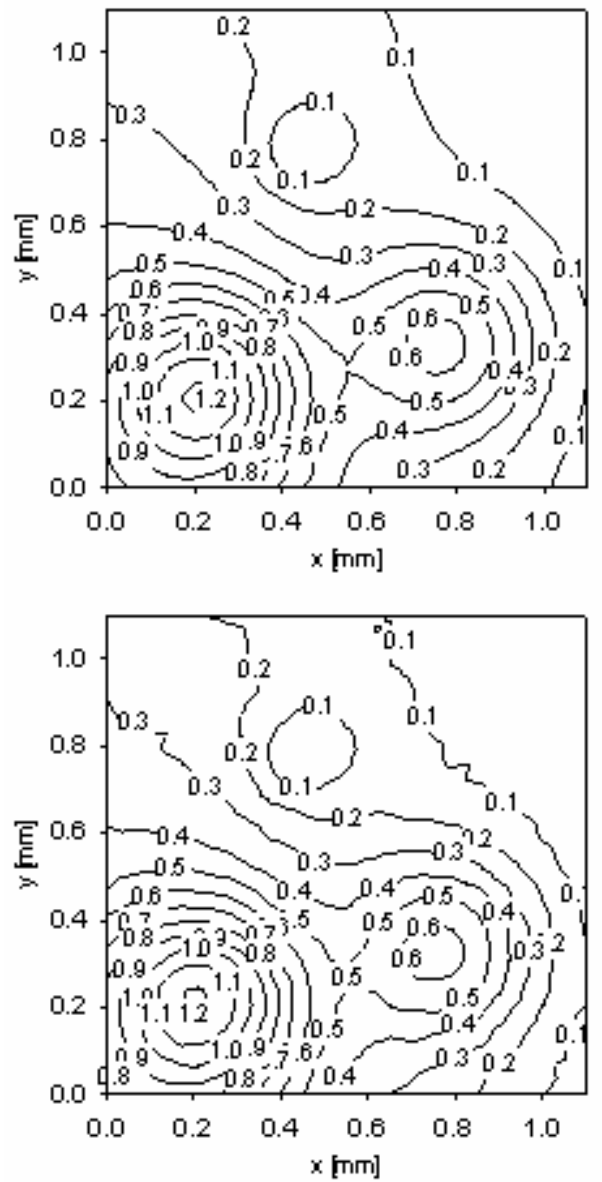

\section{CONCLUSIONS}

A comparison between modal Zernike and zonal B-Spline fitting methods has been performed for the case of simple and complex wavefront fitting. Results obtained show clearly the strengths and weaknesses of both methods when applied to different types of surfaces. In the simple wavefront case, the Zernike basis yields an excellent fit quality from degree 4 , whereas cubic B-Spline representation attains similar quality when medium-high NBP are chosen. For simple wavefronts, thus, Zernike fitting yields equivalent result with a lower computational cost.

When complex wavefronts are considered, however, the best fitting quality is achieved in the B-Spline medium-high NBP zone. The Zernike representation can not reach the B-Spline excellent fit quality as the problem becomes ill-conditioned in this case.

Once the comparison was done for theoretical wavefronts, we gained some insight into the experimental case by adding white noise to the

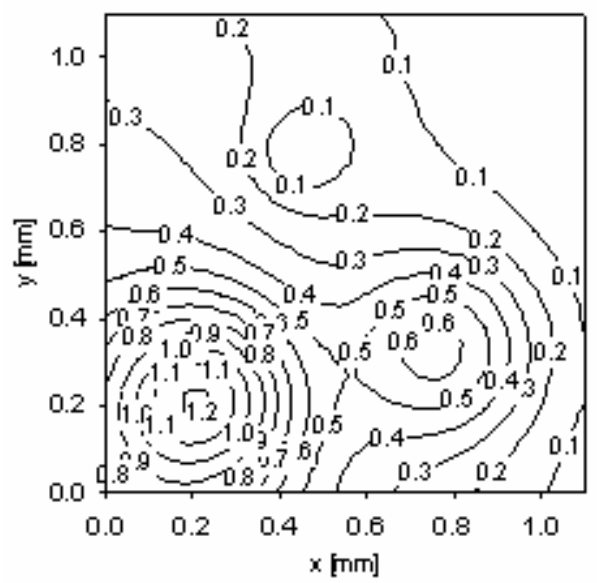
theoretical wavefronts and, for the simple wavefront case, an experimental wavefront was obtained and fitted. In all these cases, the predictions of the theoretical fits were confirmed, showing the usefulness of B-Spline fitting in complex wavefront shapes.

Fig. 8- (Up) Contour plot of the original complex wavefront, (Left-down) contour plot of the original complex wavefront plus random noise, (Right-down) contour plot of the reconstructed complex wavefront plus random noise by a NBPs= $11 \times 11$ B-Spline fitting.

\section{ACKNOWLEDGMENTS}

M.Ares would like to thank the Ministerio de Educacion y Ciencia for the PhD grant AP2003-3140 received. Also, the authors thank J.I.Garzón for his contribution to the earlier stages of the work. 


\section{REFERENCES}

1. R.R.Rammage, D.R.Neal, R.J.Copland, "Application of Shack-Hartmann wavefront sensing technology to transmissive optic metrology", Advanced Characterization Techniques for Optical, Semiconductor, and Data Storage Components, Proceedings of SPIE, Vol.4779, pp.161-172, 2002.

2. D. Malacara, "Optical Shop Testing" $2^{\text {nd }}$ Ed., Wiley, 1992.

3. W.H.Southwell, "Wave-front estimation from wave-front slope measurements", J.Opt.Soc.Am., Vol.70, pp.998-1006, August 1980.

4. K.W.Farmer, "Scattered data interpolation by $\mathrm{C}^{2}$ quintic splines using energy minimization", Thesis submitted to the Graduate Faculty of the University of Georgia in partial fulfillment of the requirements for the Degree Master of Arts, Athens-Georgia, 1997.

5. Enrique J.Fernández, Pablo Artal, "Membrane deformable mirror for adaptive optics: performance limits in visual optics", Optics Express, Vol.11, No9, pp. 1056-1069, 2003.

6. Pedro M.Prieto, Enrique J.Fernández, Silvestre Manzanera, Pablo Artal, "Adaptive optics with a programmable phase modulator: applications in the human eye", Optics Express, Vol.12, №17, pp.4059-4071,2004.

7. D. F. Rogers, "B-Spline Curves and Surfaces for Ship Hull Definition", Computer-Aided Hull Surface Definition Symposium, Annapolis-Maryland, 1977.

8. C.de Boor, "A Practical Guide to Splines" Rev. Ed., Springer-Verlag New York Inc., 2001.

9. Ake Björck, "Numerical Methods for Least Squares Problems", Society for Industrial and Applied Mathematics, Philadelphia, 1996.

10. C.L.Lawson, R.J.Hanson, "Solving Least Square Problems", Prentice-Hall, Englewood Cliffs, N.J., 1974

11. William H. Press, Saul A. Teukolsky, William T. Vetterling, Brian P. Flannery, "Numerical Recipes in C" $2^{\text {nd }}$ Ed., Cambridge University Press, 1997. 